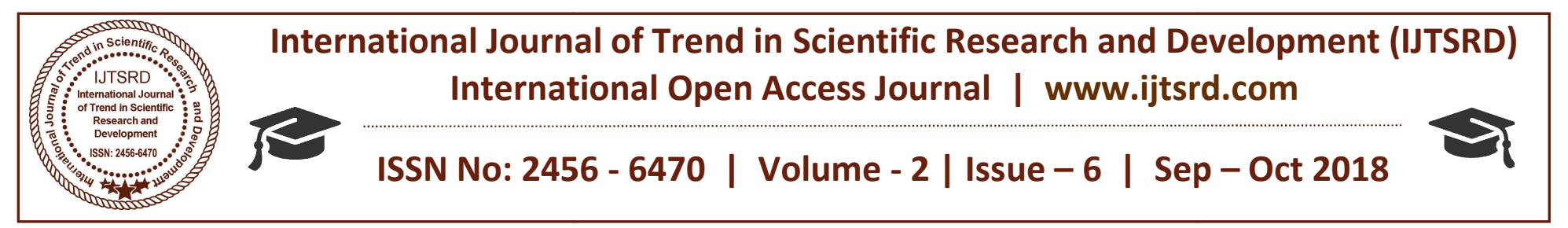

\title{
Design and Development of Honeycomb Structure for Additive Manufacturing
}

\author{
Narendra Kumar Rajak ${ }^{1}$, Prof. Amit Kaimkuriya ${ }^{2}$ \\ ${ }^{1}$ PG Scholar, ${ }^{2}$ Assisstant Professor \\ Department of Mechanical Engineering, Millennium Institute of Technology, Bhopal, India
}

\section{ABSTRACT}

the demand for shorter product development time has resulted in the introduction of a new paradigm called Additive Manufacturing (AM). Due to its significant advantages in terms of cost effective, lesser build time, elimination of expensive tooling, design flexibility AM is finding applications in many diverse fields of the industry today. One of the recent applications of this technology is for fabrication of cellular structures. Cellular structures are designed to have material where it is needed for specific applications. Compared to solid materials, these structures can provide high strength-to-weight ratio, good energy absorption characteristics and good thermal and acoustic insulation properties to aerospace, medical and engineering products. However, due to inclusion of too many design variables, the design process of these structures is a challenge task. Furthermore, polymer additive manufacturing techniques, such as fused deposition modeling (FDM) process which shows the great capability to fabricate these structures, are still facing certain process limitations in terms of support structure requirement for certain category of cellular structures. Therefore, in this research, a computeraided design (CAD) based method is proposed to design and develop hexagonal honeycomb structure (self-supporting periodic cellular structure) for FDM process. This novel methodology is found to have potential to create honeycomb cellular structures with different volume fractions successfully without any part distortion. Once designing process is complete, mechanical and microstructure properties of these structures are characterized to investigate effect of volume fraction on compressive strength of the part. Volume fraction can be defined as the volume percentage of the solid material inside the cellular structure and it is varied in this thesis by changing the cell size and wall thickness of honeycombs. Compression strength of the honeycomb structure is observed to increase with the increase in the volume fraction and this behavior is compared with an existing Wierzbicki expression, developed for predicting compression properties. Some differences are noticed in between experimentally tested and Wierzbicki model estimated compressive strength. These differences may be attributed to layer by layer deposition strategy and the residual stress inherent to the FDM-manufacturing process.

Keyword: Computer-aided design (CAD), Cellular structures, Resin Transfer Moulding (RTM), Design for Additive Manufacturing (DFAM)

\section{INTRODUCTION}

The demands for lighter, stronger, and more customizable parts have necessitated the research and development of new technologies, tools, and methodologies that can satisfy the new demands of the modern world. In this regard, the advent and continual improvement of one technology, additive manufacturing, has dramatically changed the way engineers pursue design and manufacturing. Additive manufacturing, once referred to as Rapid Prototyping (RP), has been used in many diverse field of industry for verifying the concepts (concept modeling) prior to production. However, with advancement of material science, this new and promising technology has eliminated many barriers to manufacturing and has allowed designers to achieve a level of complexity and customizability that is infeasible using traditional machining processes. As a result, most of the industries like Siemens, Phonak, Widex, Boeing and Airbus are now using this technology for producing their functional parts that are used in the final 
products. One such application of this technology is for manufacturing of customized, lightweight cellular structures. They have several advantages such as high strength-to-weight ratio and strong thermal and acoustic insulation properties. These types of structures are suitable for any weight-critical applications, particularly in the aerospace and automotive industries. This research will present a method for the design of these cellular structures for mold making application.

Additive manufacturing (AM) is an additive fabrication process where a three-dimensional part is produced by stacking layers of thin 2-D cross sectional slices of materials one over another without use of tooling and human intervention. The process begins with a solid model CAD drawing of the object. The CAD model is then converted in to .STL file format and sent to an AM machine for prototype building [1]. The whole process of design to physical model through various intermediate interfacing stages is shown in Fig. 1. These steps are common to most AM systems but the mechanisms by which the individual layers are created depend on the specific system.

Currently, many technologies exist that into the broad definition of AM. These technologies are supported by various distinct process categories. These are: photo polymerization, powder bed fusion, extrusionbased systems, printing, sheet lamination, beam deposition, and direct write technologies $[2,3]$. Each of these processes has its own distinct set of advantages and disadvantages regarding characteristics such as surface finish, manufacturing speed and layer resolution. Of these different processes, three technologies are most commonly used: fused deposition modeling (FDM), stereo lithography (SLA) and selective laser sintering (SLS). These three processes will be briefly outlined in the following sections.

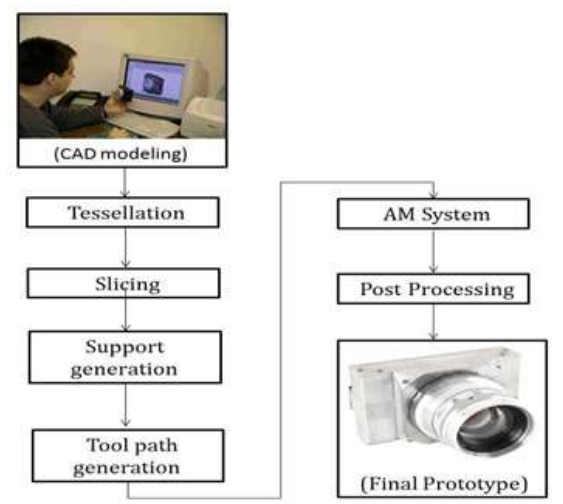

Figure 1: CAD-Prototype intermediate stages
Fused Deposition Modelling (FDM) was introduced and commercialized by Stratasys, Minnesota, USA in 1991.FDM process builds prototype by extruding material (normally thermoplastic like ABS) through a nozzle that traverses in $\mathrm{X}$ and $\mathrm{Y}$ to create each two dimensional layer. As each layer is extruded, it bonds to the previous layer and solidify. The platform is then lowered relative to the nozzle and the next slice of the part is deposited on top of the previous slice. A second nozzle is used to extrude a different material in order to build-up support structures for the part where needed.

\section{LITERATURE SURVEY}

Yu and Li et al.[1991] This paper proposes to use solid offset to cut down the solid volume to be built. The background theory for obtaining the reducedvolume solid is negatively offsetting the CSG The approach is applicable to solids defined by constructive solid geometry (CSG)

Ganesan and Fadel et al [1993] A simple effective method is presented here for creating (outside of the solid modeler) hollow CAD models of the object using offsetting techniques. This method is not suitable for creating hollow parts that have varying surface normals.

Koc and Lee et al. [1993] This paper presents a new method of using non-uniform offsetting and biarcs fitting to hollow out solid objects or thick walls to speed up the part building processes on rapid prototyping (RP) systems. Offset STL model contains some triangular facets with overlaps and inconsistent orientations.

Qu and stucker et al [1994] This paper presents a new 3D offset method for modifying CAD model data in the STL format. In this method, vertices, instead of facets, are offset. The magnitude and direction of each vertex offset is calculated using the weighted sum of the normals of the facets that are connected to each vertex.

Liu and Chen et al.[1996] presented to meet the demands of hollowed prototypes in casting and rapid prototype manufacturing. Offsetting along the Z-axis and cross sectional contour offsetting are employed to perform the hollowing operation.

Dutra et al et al.[1998] measured the stiffness of $\mathrm{Ti}-$ $6 \mathrm{Al}-4 \mathrm{~V}$ open cellular foams fabricated by electron 
beam melting (EBM). Results are found to be in good agreement with the Gibson-Ashby model for open cellular foam materials Wang and McDowell have performed a comprehensive review of analytical modeling, mechanics, and characteristics of various metal honeycombs.

Garcia et al [1999] CNTs were grown on alumina fiber cloth. These fibers were used as reinforcements in matrix material. The growth of CNTs led to an increase in inter-laminar shear properties of the order of $69 \%$ as compared to alumina cloth composite the elastic properties of FFRC (Fuzzy fiber reinforced composite) using mechanics of materials approach and Mori-Tanaka method considering with and without the interphase between $\mathrm{CNT}$ and polymer.

Johnson et al. [2000] provided a more comprehensive analytical model of the truss structure by considering each strut as a beam experiencing axial, bending, shearing, and torsion effects. He analyzed the octet-truss structure inside finite-element environment using a unit-truss model that consists of a node and set of half-struts connecting to the node.

Chang et. al. [2005] A standard thermosetting liquid resin, with the commercial brand name Quires 406 PA is used in the matrix in the form of orthophthalic unsaturated polyester (UP). It is acquired from the company MR-Dinis dos Santos (Lisbon, Portugal) and its characteristics are presented in the APPENDIX B.

Neshumayevet. al. [2006] RTM processes are capable to manufacture high geometrical complex parts and due to the comparably low cost of the raw materials and preforming technologies, it is applicable for medium size manufacturing series. However, for successful composite fabrication in RTM process, proper mold design should be done prior to processing. Moreover when mold is in complex in nature.

\section{PROBLEM IDENTIFICATION RESEARCH OBJECTIVE}

The basic problem definition is as follows

$>$ Design of cellular solid is often a difficult task using existing CAD packages due to the level of complexity associated with it.

$>$ Designed by FDM is tested for RTM application.

$>$ The complete process should be integrated with the existing CAD platform
The main research objectives is as follows

$>$ Generate and design periodic cellular structures e.g. honeycomb shaped

$>$ Whole program is automated using VB script programming and is validated for many complex shaped parts.

\section{METHODOLOGY}

In order to understand optimization of structures, the definitions of three categories of structural optimization are explained below. Literature reveals that the optimization of part geometry and topology of the structural lay-out has a great impact on the performance of the structures.

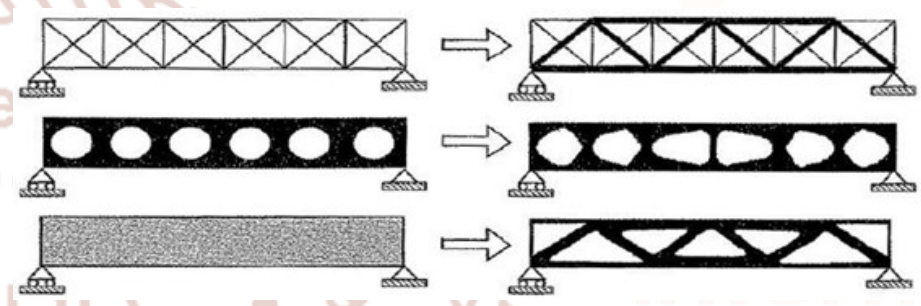

Figure 2: Size (top), shape (middle) and topology (bottom) optimization

A typical size optimization involves finding the optimal cross-sectional area of each strut in a truss structure. Shape optimization computes the optimal form that defined by the boundary curves or boundary surfaces of the body. The process may involve moving nodes to change the shape of the structure; however, the element-node connectivity remains intact. According to Rozvany, topology optimization can be defined as determining the optimal connective sequences of members or elements in a structure. It consists of both size and shape optimization and has been used most frequently by design engineers to optimize their part structures for AM application.

The topology optimization techniques used by the design are based on one of two approaches: the homogenization (continuum) approach and the ground (discrete) truss approach. By using some continuous \& variables such as cross-sectional area, void sizes, these two approaches transform the discrete problem into a continuous one. The details of these two approaches are discussed in.

Though structural optimization approach is used for the design, there is always a need for actual optimization routine. There are many different optimization algorithms depending on the applications such as mathematical programming techniques, 
stochastic process techniques, and statistical methods. According to Rozvany and Zhou, these algorithms fall in two categories: direct methods and indirect method. Direct methods (mathematical programming) consist of iteratively calculating the value of the objective function, its gradient with respect to all the design variables, and a change of design variables resulting in cost reduction until the local minimum of the objective function is found. These methods are very robust, however, the calculation of gradients can be time-taking process, and sometimes can only optimize a limited number of design variables.

On the other hand, indirect methods, such as optimality criterion, attempt to satisfy some design criteria of the structure instead of optimizing the main objective function. In many cases, such as uniform stresses, it has been found that direct method provides the same solutions as that of indirect methods. Chu et al. compared the performance of three methods namely, Particle Swam Optimization (PSO), Levenberg-Marquardt (LM) and Active-set
Programming while designing octet lattice cellular structures to achieve desired strength and stiffness. Results show that LM is more efficient algorithms for this class of problems.

Selection of an appropriate process requires addressing to various criteria such as cost, part quality, part properties, build envelope, build time (speed) and other concerns suiting to a particular situation. A number of studies have been carried out in this direction, predominantly concerning with the development of decision support systems for assisting AM users in selecting the most appropriate AM process.

\section{RESULT AND ANALYSIS}

In this section, a comparative benchmarking analysis between three infill patterns of the mould such as solid, sparse and honeycomb is presented. It will be interesting to know their material count and build time. Table 1 shows these values along with their respective build times and weights.

Table 1: RTM mould characterization with three different infill patterns

\begin{tabular}{|c|c|c|c|c|}
\hline Infill Pattern & RTM mould & Model Material $\left(\mathrm{cc}^{3}\right)$ & Build Time (hr: min) & Weight (gram) \\
\hline \multirow{3}{*}{ Solid } & Bottom & Of $\mid$ |r $169.66 \mathrm{\| n} \mathrm{SC}$ & lentitil 7.42 & \multirow{3}{*}{333} \\
\hline & Top & 166.77 & 7.29 & \\
\hline & Total & 336.43 & 14.71 & \\
\hline \multirow{3}{*}{ Sparse } & Bottom & 53.96 & 3.52 & \multirow{3}{*}{102} \\
\hline & Top & 50.71 & 4.05 & \\
\hline & Total & 104.67 & 7.57 ๑ & \\
\hline \multirow{3}{*}{ Honeycomb } & Bottom & 111.59 & 13.25 & \multirow{3}{*}{228} \\
\hline & Top & 71.12 & 11.44 & \\
\hline & Total & 182.71 & 24.69 & \\
\hline
\end{tabular}

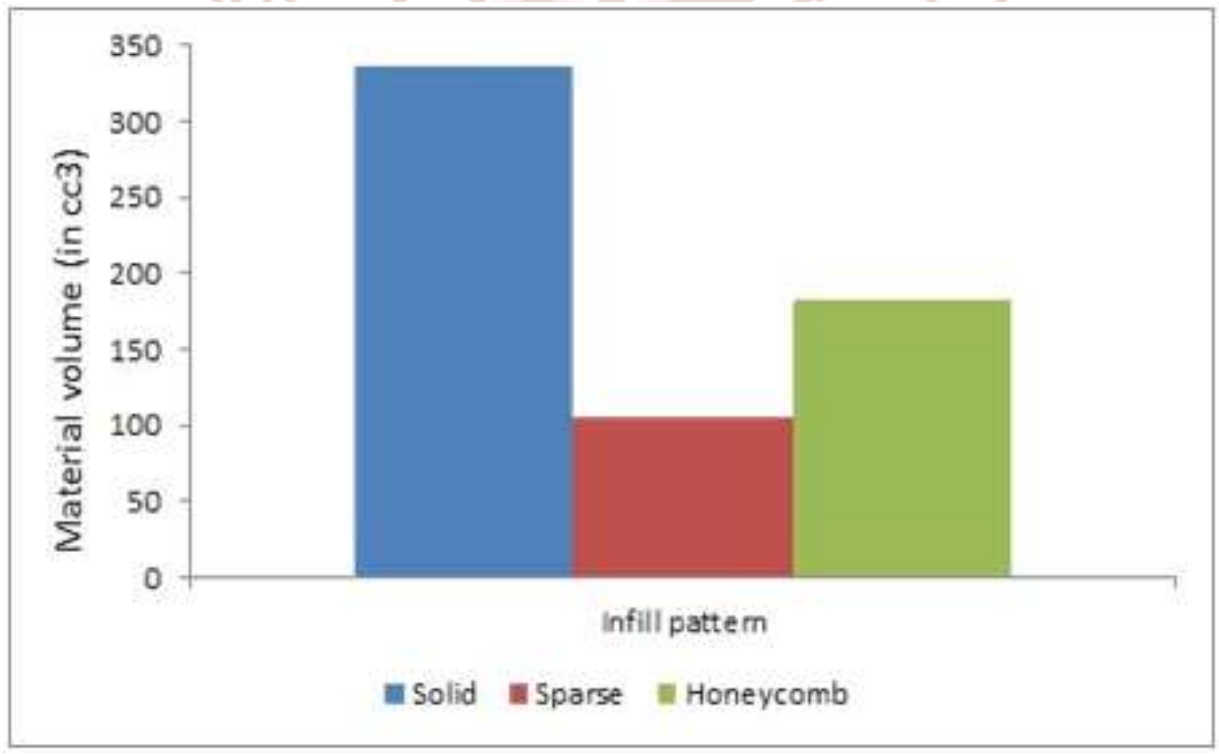

Figure 3: Trade-off between build time and infill pattern 
From the above table, it is clear that solid mould is consuming more material followed by honeycomb and sparse. The time taken to print these moulds is also found to be in the same order as of material. It is also noted that though material and build time is minimum for sparse mould it failed during testing due to insufficient resistance to the applied force, whereas honeycomb mould is able to resist the load without any deformation. In this regard, direct 30\% weight reduction is achieved which in turn will reduce the mould fabrication cost. For a clear understanding, bar graphs are also presented displaying the material consumption and build time consumed by these moulds.

\section{CONCLUSIONS AND FUTURE WORK}

FDM is a promising technology that builds part of any geometry by sequential deposition of the material extruded out from the tip of a nozzle in a temperature controlled environment. It has significant advantages in terms of elimination of expensive tooling, flexibility, and possibility of producing complex parts and shapes. One of the current challenges faced by FDM users relates to the cost of prototype and this is partly attributed to expensive process-able material. In this direction the present work emphasizes on the improving material distribution by introducing cellular solids since they offer significantly high strength at low relative mass.

DFM is a useful approach to reduce, and hopefully minimize, manufacturing and assembly difficulties and costs. This engineering technique focuses not only on the design of a part but also on its producibility. The ease of producing a part with a manufacturing process can dramatically reduce its manufacturing costs. Producibility is not only affected by the manufacturing process but also by the geometry of the part, and many other properties of the part. To take advantage of this approach, it is necessary to identify their (additive manufacturing processes) specific manufacturing capabilities as well as their manufacturing constraints that must be respected.

The need for minimizing the expensive build materials, fabrication time, energy consumption motivated the researchers towards designing cellular structures since a key advantage offered by these structure is high strength accompanied by a relatively low mass. However due to their complexity it is often difficult to generate using conventional manufacturing process. In this regard, advances in AM and CAD systems have allowed for the creation of From mechanical characterization analysis, it is found that compressive strength of honeycomb is independent of height and cell size rather dependent upon their relative density. Relative density or volume fraction is defined as the volume percentage of the solid material in the cellular structure.

It can be varied by controlling cell size and wall thickness. An increase in wall thickness and decrease in cell size, results increase in part density as well as relative density. It is also noticed that compressive strength of the cellular structure also increases with increase in relative density. This increasing trend is later confirmed with Gibson-Ashby model with some deviation in the plot. The deviation may be attributed to the residual stress and layer by layer deposition strategy of AM process.

In this research, only hexagonal honeycomb, designed by FDM is tested for RTM application. In future, other category of cellular structure such as truss structure should be investigated to study their properties. Though honeycomb cellular structure is designed, it's optimization is not carried out yet for the given loading condition. It should be taken in to consideration for generating optimal part design. Tool-path optimization should be in future in order to reduce build time of the honeycomb structure which will enable low cost FDM products. In addition, the complete process should be integrated with the existing CAD platform, through an add-on installation, for quick and an easy part generation.

\section{References}

1. S. Upcraft and R. Fletcher, "The Rapid Prototyping Technologies," Assembly Automation, vol. 23(4), pp. 318-330, 2003

2. C. C. Kai and L. K Fai, "Rapid Prototyping: Principles and Applications in Manufacturing," John Wiley and Sons, Singapore, 1997

3. S.S. Mahapatra and B. N. Panda, "Benchmarking of rapid prototyping systems using grey relational analysis," Int. J. Services and Operations Management, Vol. 16, No. 4, pp.460-477, 2013

4. R. Noorani, "Rapid Prototyping-Principles and Application," John Wiley \& Sons, New Jersey, USA, 2005

5. S. H. Masood and W. Q. Song, "Development of New Metal / Polymer Materials for Rapid Tooling 
using Fused Deposition Modelling," Materials \& Design, vol. 25, pp. 587-594, 2004.

6. "Image: FDM Process Flow," ed. http://chuansong.me/n/492448

7. http://www.stratasys.com/industries/automotive

8. P. F. Jabobs, "Rapid Prototyping \& Manufacturing: Fundamentals of Stereo lithography," TX: McGraw-Hill, 1993.

9. "Image: Siemens hearing aid," ed. http://www.medical.siemens.com.

10. "Image: Invisalign braces," ed. http://www.invisalign.com.

11. D. W. Rosen, I. Gibson, and B. Stucker, "Additive Manufacturing Technologies," Springer, 2010.

12. J. Wooten, "Aeronautical case studies using rapid manufacture, in Rapid Manufacturing (Hopkinson, N., Hague, R., and Dickens, P., eds.)," ch. 15, John Wiley \& Sons, 2006

13. "Image: Propellor,"

Ed. http://www.steg.com.au/capabilities/additive manufacturing/metals/

14. Vayre, Benjamin, F. Vignat, and F. Villeneuve, "Identification on some design key parameters for additive manufacturing: application on Electron Beam Melting," Procedia CIRP, Vol.7, pp.264269, 2013.

15. www.amrc.co.uk/wpcontent/uploads/AMRC_DPG_CircuitBracket.pdf

16. http://www.eos.info/eos_airbusgroupinnovationtea m_aerospace_sustainability study

17. http://www.geaviation.com/company/additivemanufacturing.html

18. Yan, Chunze, et al. "Evaluations of cellular lattice structures manufactured using selective laser melting." International Journal of Machine Tools and Manufacture, vol.62,pp. 32-38, 2012.

19. "Image: Honeycomb," ed. http://www.ec21.com/offer_detail/Sell_SSH301_and_SSH-304_Stainless--23593211.html.

20. "Image: Foam," ed. http://www.zhongweiaf.com/en/

21. V. S. Deshpande, N. A. Fleck, and M. F. Ashby, "Effective properties of the octet-truss lattice material," Journal of Mechanics and Physics of Solids, vol. 49, pp. 1747-1769, 2001
22. M. F. Ashby, A. G. Evans, N. A. Fleck, L. J. Gibson, J. W. Hutchinson, and H. N. G. Wadley, Metals Foams: A Design Guide. Woburn, MA: Butterworth-Heinemann, 2000.

23. J. Chu, S. Engelbrecht, G. Graf, and D. W. Rosen, "A comparison of synthesis methods for cellular structures with application to additive manufacturing," Rapid Prototyping Journal, vol. 16, pp. 275-283, 2010.

24. K.M. Yu and C.L. Li, "Speeding up rapid prototyping by offset, Proceedings of the Institute of Mechanical Engineers," Part B: Journal of Engineering Manufacturing, Vol. 209, pp.1-8, 1995.

25. M. Ganesan, and G. M Fadel, "Hollowing rapid prototyping parts using offsetting techniques," Proceedings of the 5th International Conference on Rapid Prototyping, June 1994, University of Dayton Research Institute, Dayton, 1994.

26. [26] B. Koc, and Y. S. Lee, "Non-uniform offsetting and hollowing objects by using biarcs fitting for rapid prototyping processes," Computers in Industry, Vol. 47, pp. 1-23, 2002.

27. [27]X. Qu and B, Stucker, "A 3D surface offset method for STL-format models," Rapid Prototyping Journal, Vol.9 (3), pp.133-141, 2003.

28. Park, and C. Sang, Hollowing objects with uniform wall thickness," Computer-Aided Design, Vol.37, pp.451-460, 2005

29. Z. Zhengyu, D. Yucheng and H. Jun, A new hollowing process for rapid prototype models," Rapid Prototyping Journal, Vol. 10(3), pp. 166175,2004

30. L. E. Murr, et al. "Characterization of Ti-6Al-4V open cellular foams fabricated by additive manufacturing using electron beam melting." Materials Science and Engineering: Vol. A 527.7, pp.1861-1868, 2010 